\title{
On a strengthened version of Hardy's inequality
}

\author{
Qian $\mathrm{Xu}^{1 *}$, Meixiu Zhou ${ }^{2}$ and Xiaoming Zhang ${ }^{3}$
}

\section{"Correspondence:}

xq32153215@yahoo.com.cn

1 Jiaxing Radio \& TV University, Jiaxing, Zhejiang 314000 , P.R. China

Full list of author information is

available at the end of the article

\section{Abstract \\ On the basis of analytic method and skill, a strengthened version of well-known Hardy's inequality is obtained. In comparison with some similar results given in recent decades, it successfully reduced the related coefficient.}

MSC: 26D15

Keywords: inequality; Hardy's inequality; convex function; Hadamard's inequality

\section{Introduction}

There are many applications with well-known Hardy's inequality in analytics, which refers to the following: let $a_{k}>0(k=1,2, \ldots), p>1$, then

$$
\left(\frac{p}{p-1}\right)^{p} \sum_{n=1}^{\infty} a_{n}^{p}>\sum_{n=1}^{\infty}\left(\frac{1}{n} \sum_{k=1}^{n} a_{k}\right)^{p}
$$

In recent decades, there have also been many results due to the extension and refinement of this inequality ( $c f$. [1-5]), especially the monograph [6], which summarized part of the research done before 2005. In research on the coefficient of (1.1), the following conclusion in the case $p=2$ was drawn in [6]:

$$
4 \sum_{n=1}^{\infty}\left(1-\frac{1}{3 \sqrt{n}+5}\right) a_{n}^{2}>\sum_{n=1}^{\infty}\left(\frac{1}{n} \sum_{k=1}^{n} a_{k}\right)^{2} .
$$

In [7], by using the method of weight-coefficient, the following inequality is proved with $p \in\left[\frac{7}{6}, 2\right]:$

$$
\left(\frac{p}{p-1}\right)^{p} \sum_{n=1}^{\infty}\left(1-\frac{15}{196} \frac{1}{n^{1-1 / p}+3,436}\right) a_{n}^{p}>\sum_{n=1}^{\infty}\left(\frac{1}{n} \sum_{k=1}^{n} a_{k}\right)^{p} .
$$

In the following section, let $p>1$ and

$$
Z_{p}= \begin{cases}p-1-\frac{(p-1)^{2}}{p} 2^{\frac{1}{p}}, & 1<p \leq 2, \\ 1-\left(\frac{p-1}{p}\right)^{p-1} 2^{\frac{p-1}{p}}, & p>2 .\end{cases}
$$

2012 Xu et al.; licensee Springer. This is an Open Access article distributed under the terms of the Creative Commons Attribution License (http://creativecommons.org/licenses/by/2.0), which permits unrestricted use, distribution, and reproduction in any medium, provided the original work is properly cited. 
We shall strengthen Hardy's inequality to

$$
\left(\frac{p-1}{p}\right)^{p} \sum_{n=1}^{\infty}\left(1-\frac{Z_{p}}{2\left(n-\frac{1}{2}\right)^{1-\frac{1}{p}}}\right) a_{n}^{p}>\sum_{n=1}^{\infty}\left(\frac{1}{n} \sum_{k=1}^{n} a_{k}\right)^{p} .
$$

\section{Relevant lemmas}

Some characters of a convex function will be cited in this section.

Definition 2.1 $L \subseteq \mathbb{R}$ is an interval, and $f: I \rightarrow \mathbb{R}$ is continuous. If

$$
f\left(\frac{x+y}{2}\right) \leq(\geq) \frac{f(x)+f(y)}{2}
$$

holds for all $x, y \in I$, then $f$ is called a convex (concave) function.

The sufficient and necessary condition for a second-order differential function $f$ to be convex (concave) function is that $f^{\prime \prime}(x) \geq(\leq) 0$ always holds for any $x \in I$. The famous Hadamard inequality is as follows. Let $f$ be a convex (concave) function on $[a, b]$, then the equality

$$
f\left(\frac{b+a}{2}\right) \leq(\geq) \frac{1}{b-a} \int_{a}^{b} f(x) d x \leq(\geq) \frac{f(b)+f(a)}{2}
$$

holds if and only if $f$ is a linear function.

Lemma 2.1 Let $p>1$, and $Z_{p}$ is defined in the first section, then $0<Z_{p}<\frac{1}{2}$.

Proof The proof includes two parts.

Part 1: When $1<p \leq 2$, if we can obtain $f(p):=2^{-\frac{1}{p}}-1+\frac{1}{p}>0$, then we can get $Z_{p}>0$ obviously. Since

$$
f^{\prime}(p)=\frac{1}{p^{2}} 2^{-\frac{1}{p}} \ln 2-\frac{1}{p^{2}}=\frac{1}{p^{2}} 2^{-\frac{1}{p}}\left(\ln 2-2^{\frac{1}{p}}\right)<0,
$$

$f(p)$ is monotone decreasing on $(1,2]$, then $f(p) \geq f(2)=\frac{\sqrt{2}}{2}-\frac{1}{2}>0$.

Meanwhile, $Z_{p}<\frac{1}{2}$ is equivalent to

$$
p-\frac{3}{2}<\frac{(p-1)^{2}}{p} \cdot 2^{\frac{1}{p}}, \quad-\frac{3}{2}<\left(p-2+\frac{1}{p}\right) \cdot 2^{\frac{1}{p}}-p .
$$

The above two inequalities obviously hold with $1<p<\frac{3}{2}$. If $\frac{3}{2}<p<2$, then $p-2+\frac{1}{p}$ is increasing about $p, 2^{\frac{1}{p}}$ and $-p$ are decreasing in relation to $\mathrm{p}$. Then

$$
\begin{aligned}
& \left(p-2+\frac{1}{p}\right) \cdot 2^{\frac{1}{p}}-p>\left(\frac{3}{2}-2+\frac{1}{\frac{3}{2}}\right) \cdot 2^{\frac{1}{8}}-\frac{13}{8}>-\frac{3}{2}, \quad \frac{3}{2}<p<\frac{13}{8} \\
& \left(p-2+\frac{1}{p}\right) \cdot 2^{\frac{1}{p}}-p>\left(\frac{13}{8}-2+\frac{1}{\frac{13}{8}}\right) \cdot 2^{\frac{1}{7}}-\frac{7}{4}>-\frac{3}{2}, \quad \frac{13}{8}<p<\frac{7}{4}
\end{aligned}
$$




$$
\begin{aligned}
& \left(p-2+\frac{1}{p}\right) \cdot 2^{\frac{1}{p}}-p>\left(\frac{7}{4}-2+\frac{1}{\frac{7}{4}}\right) \cdot 2^{\frac{1}{15}}-\frac{15}{8}>-\frac{3}{2}, \quad \frac{7}{4}<p<\frac{15}{8} \\
& \left(p-2+\frac{1}{p}\right) \cdot 2^{\frac{1}{p}}-p>\left(\frac{15}{8}-2+\frac{1}{\frac{15}{8}}\right) \cdot 2^{\frac{1}{2}}-2>-\frac{3}{2}, \quad \frac{15}{8}<p<2 .
\end{aligned}
$$

Part 2: When $p>2$, then it should be proved that $\left(\frac{p-1}{p}\right)^{p-1} 2^{\frac{p-1}{p}}<1$ and $\left(\frac{p-1}{p}\right)^{p-1} 2^{\frac{p-1}{p}}>\frac{1}{2}$ are respectively equivalent to $2<\left(1+\frac{1}{p-1}\right)^{p}$ and $\left(1+\frac{1}{p-1}\right)^{p}<2^{2+\frac{1}{p-1}}$. Since $\left(1+\frac{1}{p-1}\right)^{p}$ is strictly decreasing for $p \in(2,+\infty)$, then it is proved that

$$
\left(1+\frac{1}{p-1}\right)^{p}>\lim _{p \rightarrow \infty}\left(1+\frac{1}{p-1}\right)^{p}=e>2
$$

and

$$
\left(1+\frac{1}{p-1}\right)^{p}<\left(1+\frac{1}{2-1}\right)^{2}=4<2^{2+\frac{1}{p-1}}
$$

The proof of Lemma 2.1 is completed.

\section{Lemma 2.2}

(i) Let $x \geq 1, p>2$, then

$$
\left(1-Z_{p} x^{\frac{1}{p}-1}\right)^{\frac{2-p}{p-1}}\left(1-\frac{p-2}{p-1} Z_{p} x^{\frac{1}{p}-1}\right)>1 .
$$

(ii) Let $p>1$, then $f: x \in[1,+\infty) \rightarrow x^{\frac{1}{p}-2}-Z_{p} x^{\frac{2}{p}-3}$ is a convex function.

Proof (i) The proposition is equivalent to

$$
1-\frac{p-2}{p-1} Z_{p} x^{\frac{1}{p}-1}>\left(1-Z_{p} x^{\frac{1}{p}-1}\right)^{\frac{p-2}{p-1}} .
$$

Bernoulli's inequality refers to the following: $(1+t)^{\alpha} \leq 1+\alpha t(t>-1,0<\alpha<1)$ holds if $t=0$. If $t=-Z_{p} x^{-1+\frac{1}{p}}, \alpha=\frac{p-2}{p-1}$, then formula (2.2) holds.

(ii)

$$
\begin{aligned}
f^{\prime \prime}(x) & =\left(\frac{1}{p}-2\right)\left(\frac{1}{p}-3\right) x^{\frac{1}{p}-4}-Z_{p}\left(\frac{2}{p}-3\right)\left(\frac{2}{p}-4\right) x^{\frac{2}{p}-5} \\
& =\frac{(2 p-1) x^{\frac{2}{p}-5}}{p^{2}}\left[(3 p-1) x^{1-\frac{1}{p}}-2 Z_{p}(3 p-2)\right] \\
& \geq \frac{(2 p-1) x^{\frac{2}{p}-5}}{p^{2}}\left[(3 p-1)-2 Z_{p}(3 p-2)\right] .
\end{aligned}
$$

According to Lemma 2.1,

$$
f^{\prime \prime}(x) \geq \frac{(2 p-1) x^{\frac{2}{p}-5}}{p^{2}}[(3 p-1)-(3 p-2)]>0,
$$

so, $f$ is a convex function. The proof of Lemma 2.2 is completed. 
Lemma 2.3 Let $n$ be a positive natural number, $p>1$.

(i) If $1<p \leq 2$, then

$$
\sum_{k=1}^{n} \frac{1}{\left(k-\frac{1}{2}\right)^{\frac{1}{p}}} \leq \frac{p}{p-1}\left(n^{1-\frac{1}{p}}-\frac{Z_{p}}{p-1}\right)
$$

(ii) If $p>2$, then

$$
\sum_{k=1}^{n} \frac{1}{\left(k-\frac{1}{2}\right)^{\frac{1}{p}}} \leq \frac{p}{p-1} n^{1-\frac{2}{p}}\left(n^{1-\frac{1}{p}}-Z_{p}\right)^{\frac{1}{p-1}}
$$

Proof (i) If $n=1$, inequality (2.3) is proved easily. Assume that when $n=m \geq 1$, the following equality holds for $n=m+1$ :

$$
\begin{aligned}
\sum_{k=1}^{m+1} \frac{1}{\left(k-\frac{1}{2}\right)^{\frac{1}{p}}} & \leq \frac{p}{p-1}\left(m^{1-\frac{1}{p}}-\frac{Z_{p}}{p-1}\right)+\frac{1}{\left(m+\frac{1}{2}\right)^{\frac{1}{p}}} \\
& =\frac{p}{p-1}\left[(m+1)^{1-\frac{1}{p}}-\frac{Z_{p}}{p-1}\right]-\int_{m}^{m+1} x^{-\frac{1}{p}} d x+\frac{1}{\left(m+\frac{1}{2}\right)^{\frac{1}{p}}}
\end{aligned}
$$

Because $x^{-\frac{1}{p}}$ is a convex function on [ $\left.m, m+1\right]$, then according to Hadamard's inequality of a convex function, formula (2.3) also holds if $n=m+1$.

(ii) If $n=1$, inequality (2.4) is proved easily. Assume that when $n=m \geq 1$, the inequality holds. For $n=m+1$,

$$
\begin{aligned}
\sum_{k=1}^{m+1} \frac{1}{\left(k-\frac{1}{2}\right)^{\frac{1}{p}} \leq} & \frac{p}{p-1} m^{1-\frac{2}{p}}\left(m^{1-\frac{1}{p}}-Z_{p}\right)^{\frac{1}{p-1}}+\frac{1}{\left(m+\frac{1}{2}\right)^{\frac{1}{p}}} \\
= & \frac{p}{p-1}(m+1)^{1-\frac{2}{p}}\left((m+1)^{1-\frac{1}{p}}-Z_{p}\right)^{\frac{1}{p-1}} \\
& -\int_{m}^{m+1}\left[\frac{p}{p-1} x^{1-\frac{2}{p}}\left(x^{1-\frac{1}{p}}-Z_{p}\right)^{\frac{1}{p-1}}\right]^{\prime} d x+\frac{1}{\left(m+\frac{1}{2}\right)^{\frac{1}{p}}} \\
= & \frac{p}{p-1}(m+1)^{1-\frac{2}{p}}\left((m+1)^{1-\frac{1}{p}}-Z_{p}\right)^{\frac{1}{p-1}}+\frac{1}{\left(m+\frac{1}{2}\right)^{\frac{1}{p}}} \\
& -\frac{p}{p-1} \int_{m}^{m+1}\left[\left(1-\frac{2}{p}\right) x^{-\frac{2}{p}}\left(x^{1-\frac{1}{p}}-Z_{p}\right)^{\frac{1}{p-1}}\right. \\
& \left.+\frac{1}{p-1}\left(1-\frac{1}{p}\right) x^{1-\frac{2}{p}}\left(x^{1-\frac{1}{p}}-Z_{p}\right)^{\frac{1}{p-1}-1} \cdot x^{-\frac{1}{p}}\right] d x \\
= & \frac{p}{p-1}(m+1)^{1-\frac{2}{p}}\left((m+1)^{1-\frac{1}{p}}-Z_{p}\right)^{\frac{1}{p-1}}+\frac{1}{\left(m+\frac{1}{2}\right)^{\frac{1}{p}}} \\
& -\frac{p}{p-1} \int_{m}^{m+1} x^{-\frac{2}{p}}\left(x^{1-\frac{1}{p}}-Z_{p}\right)^{\frac{2-p}{p-1}} \\
& \times\left[\left(1-\frac{1}{p}\right) x^{1-\frac{1}{p}}-\left(1-\frac{2}{p}\right) Z_{p}\right] d x
\end{aligned}
$$




$$
\begin{aligned}
= & \frac{p}{p-1}(m+1)^{1-\frac{2}{p}}\left((m+1)^{1-\frac{1}{p}}-Z_{p}\right)^{\frac{1}{p-1}}+\frac{1}{\left(m+\frac{1}{2}\right)^{\frac{1}{p}}} \\
& -\int_{m}^{m+1} x^{-\frac{1}{p}}\left(1-Z_{p} x^{-1+\frac{1}{p}}\right)^{\frac{2-p}{p-1}}\left[1-\frac{p-2}{p-1} Z_{p} x^{-1+\frac{1}{p}}\right] d x .
\end{aligned}
$$

By inequality (2.2) and the fact that $x^{-\frac{1}{p}}$ is a convex function on $[m, m+1]$, we have

$$
\begin{aligned}
\sum_{k=1}^{m+1} \frac{1}{\left(k-\frac{1}{2}\right)^{\frac{1}{p}}} & <\frac{p}{p-1}(m+1)^{1-\frac{2}{p}}\left((m+1)^{1-\frac{1}{p}}-Z_{p}\right)^{\frac{1}{p-1}}+\frac{1}{\left(m+\frac{1}{2}\right)^{\frac{1}{p}}}-\int_{m}^{m+1} x^{-\frac{1}{p}} d x \\
& \leq \frac{p}{p-1}(m+1)^{1-\frac{2}{p}}\left((m+1)^{1-\frac{1}{p}}-Z_{p}\right)^{\frac{1}{p-1}}+\frac{1}{\left(m+\frac{1}{2}\right)^{\frac{1}{p}}}-\left(m+\frac{1}{2}\right)^{-\frac{1}{p}} \\
& =\frac{p}{p-1}(m+1)^{1-\frac{2}{p}}\left((m+1)^{1-\frac{1}{p}}-Z_{p}\right)^{\frac{1}{p-1}} .
\end{aligned}
$$

Thus, the inequality (2.4) also holds if $n=m+1$.

Lemma 2.4 Let $i$ be any positive natural number and $p>1$, then

$$
\sum_{n=i}^{\infty}\left(n^{-2+\frac{1}{p}}-Z_{p} n^{-3+\frac{2}{p}}\right)<\frac{p}{p-1}\left[\left(i-\frac{1}{2}\right)^{\frac{1}{p}-1}-\frac{Z_{p}}{2}\left(i-\frac{1}{2}\right)^{\frac{2}{p}-2}\right] .
$$

Proof Let $i=1,2, \ldots$ and

$$
f(i)=\frac{p}{p-1}\left[\left(i-\frac{1}{2}\right)^{\frac{1}{p}-1}-\frac{Z_{p}}{2}\left(i-\frac{1}{2}\right)^{\frac{2}{p}-2}\right]-\sum_{n=i}^{\infty}\left(n^{\frac{1}{p}-2}-Z_{p} n^{\frac{2}{p}-3}\right) .
$$

Then

$$
\begin{aligned}
f(i)-f(i+1)= & \frac{p}{p-1}\left[\left(i-\frac{1}{2}\right)^{\frac{1}{p}-1}-\frac{Z_{p}}{2}\left(i-\frac{1}{2}\right)^{\frac{2}{p}-2}\right]-\left(i^{\frac{1}{p}-2}-Z_{p} i^{\frac{2}{p}-3}\right) \\
& -\frac{p}{p-1}\left[\left(i+\frac{1}{2}\right)^{\frac{1}{p}-1}-\frac{Z_{p}}{2}\left(i+\frac{1}{2}\right)^{\frac{2}{p}-2}\right] \\
= & -\frac{p}{p-1} \int_{i-\frac{1}{2}}^{i+\frac{1}{2}}\left(x^{\frac{1}{p}-1}-\frac{Z_{p}}{2} x^{\frac{2}{p}-2}\right)^{\prime} d x-\left(i^{\frac{1}{p}-2}-Z_{p} i^{\frac{2}{p}-3}\right) \\
= & \int_{i-\frac{1}{2}}^{i+\frac{1}{2}}\left(x^{\frac{1}{p}-2}-Z_{p} x^{\frac{2}{p}-3}\right) d x-\left(i^{\frac{1}{p}-2}-Z_{p} i^{\frac{2}{p}-3}\right) .
\end{aligned}
$$

According to the conclusion of Lemma 2.2 and Hadamard's inequality of a convex function, the sequence $\{f(i)\}_{i=1}^{+\infty}$ is a strictly decreasing sequence. It is also known that $\lim _{i \rightarrow+\infty} f(i)=0$, then $f(i)>0$ always holds. The proof of Lemma 2.4 is completed. 
Xu et al. Journal of Inequalities and Applications 2012, 2012:300

Page 6 of 8

http://www.journalofinequalitiesandapplications.com/content/2012/1/300

3 A new strengthened version of Hardy's inequality

Theorem 3.1 Let $a_{k}>0, n \geq 1, n \in N, p>1$, and

$$
Z_{p}= \begin{cases}p-1-\frac{(p-1)^{2}}{p} 2^{\frac{1}{p}}, & 1<p \leq 2, \\ 1-\left(\frac{p-1}{p}\right)^{p-1} 2^{\frac{p-1}{p}}, & p>2,\end{cases}
$$

then

$$
\sum_{n=1}^{\infty}\left(\frac{1}{n} \sum_{k=1}^{n} a_{k}\right)^{p}<\left(\frac{p}{p-1}\right)^{p} \sum_{n=1}^{\infty}\left[1-\frac{Z_{p}}{2\left(n-\frac{1}{2}\right)^{1-\frac{1}{p}}}\right] a_{n}^{p} .
$$

Proof Let $r=\frac{p}{p-1}$, then $\frac{1}{r}+\frac{1}{p}=1$. According to Holder's inequality,

$$
\begin{aligned}
\left(\frac{1}{n} \sum_{k=1}^{n} a_{k}\right)^{p} & =\frac{1}{n^{p}}\left(\sum_{k=1}^{n}\left(k-\frac{1}{2}\right)^{-\frac{p-1}{p^{2}}}\left(k-\frac{1}{2}\right)^{\frac{p-1}{p^{2}}} a_{k}\right)^{p} \\
& \leq \frac{1}{n^{p}}\left(\sum_{k=1}^{n}\left(k-\frac{1}{2}\right)^{-\frac{p-1}{p^{2}} r}\right)^{\frac{p}{r}}\left(\sum_{k=1}^{n}\left(k-\frac{1}{2}\right)^{\frac{p-1}{p^{2}} p} a_{k}^{p}\right)^{\frac{p}{p}} \\
& =\frac{1}{n^{p}}\left(\sum_{k=1}^{n}\left(k-\frac{1}{2}\right)^{-\frac{1}{p}}\right)^{p-1} \sum_{k=1}^{n}\left(k-\frac{1}{2}\right)^{\frac{p-1}{p}} a_{k}^{p} .
\end{aligned}
$$

If $\lambda_{n}=\frac{1}{n^{p}}\left(\sum_{k=1}^{n}\left(k-\frac{1}{2}\right)^{-\frac{1}{p}}\right)^{p-1}$ and $\mu_{k}=\left(k-\frac{1}{2}\right)^{\frac{p-1}{p}}$, then

$$
\begin{aligned}
\sum_{n=1}^{\infty}\left(\frac{1}{n} \sum_{k=1}^{n} a_{k}\right)^{p} & \leq \sum_{n=1}^{\infty} \lambda_{n} \cdot \sum_{k=1}^{n} \mu_{k} a_{k}^{p} \\
& =\sum_{n=1}^{\infty}\left(\sum_{k=n}^{\infty} \lambda_{k}\right) \mu_{n} a_{n}^{p} .
\end{aligned}
$$

Thus,

$$
\sum_{k=n}^{\infty} \lambda_{k}=\sum_{k=n}^{\infty} \frac{1}{k^{p}}\left(\sum_{j=1}^{k}\left(j-\frac{1}{2}\right)^{-\frac{1}{p}}\right)^{p-1} .
$$

If $1<p \leq 2$, according to formula (2.3) and Bernoulli's inequality,

$$
\begin{aligned}
\sum_{k=n}^{\infty} \lambda_{k} & =\left(\frac{p}{p-1}\right)^{p-1} \sum_{k=n}^{\infty} \frac{1}{k^{p}}\left(k^{1-\frac{1}{p}}-\frac{Z_{p}}{p-1}\right)^{p-1} \\
& =\left(\frac{p}{p-1}\right)^{p-1} \sum_{k=n}^{\infty} k^{-2+\frac{1}{p}}\left(1-\frac{Z_{p}}{p-1} k^{-1+\frac{1}{p}}\right)^{p-1} \\
& <\left(\frac{p}{p-1}\right)^{p-1} \sum_{k=n}^{\infty} k^{-2+\frac{1}{p}}\left(1-Z_{p} k^{-1+\frac{1}{p}}\right) \\
& =\left(\frac{p}{p-1}\right)^{p-1} \sum_{k=n}^{\infty}\left(k^{-2+\frac{1}{p}}-Z_{p} k^{-3+\frac{2}{p}}\right) .
\end{aligned}
$$


If $p>2$, by using formula (2.4), we obtain

$$
\begin{aligned}
\sum_{k=n}^{\infty} \lambda_{k} & \leq\left(\frac{p}{p-1}\right)^{p-1} \sum_{k=n}^{\infty} \frac{1}{k^{p}}\left(k^{1-\frac{2}{p}}\left(k^{1-\frac{1}{p}}-Z_{p}\right)^{\frac{1}{p-1}}\right)^{p-1} \\
& =\left(\frac{p}{p-1}\right)^{p-1} \sum_{k=n}^{\infty}\left(k^{-2+\frac{1}{p}}-Z_{p} k^{-3+\frac{2}{p}}\right) .
\end{aligned}
$$

Therefore, for any $p>1$, the following inequality holds:

$$
\sum_{k=n}^{\infty} \lambda_{k} \leq\left(\frac{p}{p-1}\right)^{p-1} \sum_{k=n}^{\infty}\left(k^{-2+\frac{1}{p}}-Z_{p} k^{-3+\frac{2}{p}}\right) .
$$

By Lemma 2.4, we get

$$
\sum_{n=k}^{\infty} \lambda_{k} \leq\left(\frac{p}{p-1}\right)^{p}\left(\left(n-\frac{1}{2}\right)^{\frac{1}{p}-1}-\frac{Z_{p}}{2}\left(n-\frac{1}{2}\right)^{\frac{2}{p}-2}\right) .
$$

So, from inequalities (3.2) and (3.3), the following result can be obtained:

$$
\begin{aligned}
\sum_{n=1}^{\infty}\left(\frac{1}{n} \sum_{k=1}^{n} a_{k}\right)^{p} & \leq\left(\frac{p}{p-1}\right)^{p} \sum_{n=1}^{\infty}\left(\left(n-\frac{1}{2}\right)^{\frac{1}{p}-1}-\frac{Z_{p}}{2}\left(n-\frac{1}{2}\right)^{\frac{2}{p}-2}\right)\left(n-\frac{1}{2}\right)^{\frac{p-1}{p}} a_{n}^{p} \\
& =\left(\frac{p}{p-1}\right)^{p} \sum_{n=1}^{\infty}\left(1-\frac{Z_{p}}{2\left(n-\frac{1}{2}\right)^{1-\frac{1}{p}}}\right) a_{n}^{p} .
\end{aligned}
$$

The proof of Theorem 3.1 is completed.

\section{Competing interests}

The authors declare that they have no competing interests.

\section{Authors' contributions}

QX provided the main idea in this article and carried out the proof of the Theorem 3.1. MZ carried out the proof of Lemma 2.4. XZ carried out the proof of Lemmas 2.1-2.3. All authors read and approved the final manuscript.

\section{Author details}

${ }^{1}$ Jiaxing Radio \& TV University, Jiaxing, Zhejiang 314000, P.R. China. ${ }^{2}$ Open and distance education research institute, Zhejiang Radio \& Television University, Hangzhou, Zhejiang 310030, P.R. China. ${ }^{3}$ Zhejiang Radio \& Television University Haining College, Haining, Zhejiang 314400, P.R. China.

\section{Acknowledgements}

This research was supported by the Nature Science Foundation of the Open University of China under Grant No. Q1601E.

Received: 10 April 2012 Accepted: 28 November 2012 Published: 17 December 2012

\section{References}

1. Wedestig, A: Some new Hardy type inequalities and their limiting inequalities. J. Inequal. Pure Appl. Math. 4(3), Art. 61 (2003)

2. Bicheng, Y, Debnath, L: Generalizations of Hardy integral inequalities. Int. J. Math. Math. Sci. 22(3), 535-542 (1999)

3. Beeasck, PR: Hardy inequality and its extensions. Pac. J. Math. 11, 39-61 (1961)

4. Bicheng, Y, Zhuohua, Z, Debnath, L: On new generalizations of Hardy integral inequalities. J. Math. Anal. Appl. 217, 321-327 (1998)

5. Pachpatte, BG: Mathematical Inequalities, pp. 113-209. Elsevier, Amsterdam (2005)

6. Bicheng, Y, Yunhua, Z: An improvement on Hardy's inequality. Acta Sci. Natur. Univ. Sunyatseni 37(1), 41-44 (1998)

7. Qiling, H: On an improvement of Hardy-Hilbert's inequality in an interval. Acta Sci. Natur. Univ. Sunyatseni 39(3), 20-24 (2000) 
doi:10.1186/1029-242X-2012-300

Cite this article as: Xu et al.: On a strengthened version of Hardy's inequality. Journal of Inequalities and Applications 2012 2012:300

Submit your manuscript to a SpringerOpen ${ }^{\circ}$ journal and benefit from:

- Convenient online submission

- Rigorous peer review

- Immediate publication on acceptance

Open access: articles freely available online

- High visibility within the field

- Retaining the copyright to your article

Submit your next manuscript at $\gg$ springeropen.com 